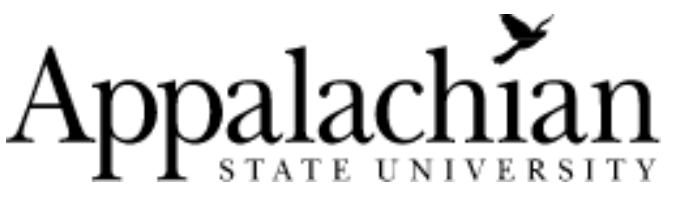

Department of Economics Working Paper

Number 11-13 | September 2011

Between the Penthouse and the

Outhouse: The Sorting of Economics

Professors

Timothy Perri

Appalachian State University 


\title{
Between the Penthouse and the Outhouse: The Sorting of Economics Professors
}

\author{
by
}

Timothy Perri*

First version November 25, 2008

This version July 22, 2011

*Department of Economics, Appalachian State University, Boone, NC, 28608. Phone: 828-262-2251; E-mail: perritj@appstate.edu 


\begin{abstract}
Oyer (2007, 2008) considered the turnover of economics professors early in their careers. He found professors are more likely to move down from higher ranked schools than up from lower ranked schools. An asymmetric information model suggests this phenomenon is explained by imperfect screening at one’s initial hiring. A smaller fraction of more able individuals and more accurate screening imply a greater chance downward movement exceeds movement up.
\end{abstract}




\section{Introduction}

In recent work, academic economists have examined their own labor market (Oyer 2006, 2007, and 2008, and Smeets et al. 2006). In a pair of papers, Oyer (2007, 2008) considers the turnover of economics professors early in their careers. Specifically, Oyer (2007) considered a sample of 1,263 economics PhDs from seven U.S. universities who went on the job market between 1979 and 1994. He tracked where these individuals were employed 10 years after their initial employment. Among his results is professors are more likely to move down from higher ranked schools than up from lower ranked schools.

One could argue it is easier to move down than up because there are more positions in lower level schools. However, this “demand” argument is not persuasive, as will be seen below. Consider the movement between 1) top 25 schools and other schools, and 2) top 50 schools and other schools. To account for “demand” elsewhere we present the number who moved up or down as a percentage of those initially in the other category.

Using Table 1 in Oyer (2007), considering top 25 schools and all others (excluding those in the missing category), we find the following.

- 44\% (132 out of 299) moved down. This represents 17\% (132 out of 792) of the number initially in the lower level.

- 4\% (28 out of 792) moved up. This represents 9\% (28 out of 299) of the number initially in the higher level. 
For top 50 schools and all others (excluding those in the missing category), we find the following.

- 40\% (157 out of 391) moved down. This represents 22\% (157 out of 700) of the number initially in the lower level.

- 5\% (34 out of 700) moved up. This represents 9\% (34 out of 391) of the number initially in the higher level.

Consider the argument is it easier to move down than up because there are more positions in lower level schools. First, plenty of openings exist at higher level schools; there are at least the ones for individuals who were terminated. Second, if higher level schools choose to hire new PhDs and not those who have been on the faculty at lower level schools, this is evidence lower level schools do not make many mistakes---do not hire many stars. Third, the evidence presented suggests, even accounting for "demand" at different levels, individuals are about twice as likely to move down as they are to move up.

In order to explain the phenomenon of more economics professors moving down than moving up, the next section considers a screening model.

\section{A screening model}

In this section, we consider why individuals are more likely to move to lower ranked schools than they are to higher ranked schools. Call higher ranked schools Type One schools, or T1s, and lower ranked schools Type Two schools, or T2s. Denote 
individual ability by $\theta$, and let individuals be either stars $(S)$ or lemons $(L)$ with respective ability levels $\theta_{S}$ and $\theta_{L}$, where $\theta_{S}>\theta_{L}>0$.

Suppose schools receive an imperfect signal of a new PhD's ability. The signal is either favorable or unfavorable. T1 schools hire only those with favorable signals; those individuals are relatively scarce, so T1s hire all of them. Those with unfavorable signals are hired by $\mathrm{T} 2$ schools $^{1}$. T2 schools cannot attract those with favorable signals. The signal of a new PhD's ability is based on all the information available at the time one is hired: the identity of one's graduate school, transcripts, reference letters, job paper, etc. ${ }^{2}$ It is assumed the probability of a favorable signal, given $\theta$, prob(favorable $\mid \theta)$, is positively related to $\theta$ in a linear fashion and equals $\lambda \theta / \theta_{S}$, with $0<\lambda \leq 1$. Thus $\operatorname{prob}($ favorable $\mid$ star $)=\lambda$, and prob(favorable $\mid$ lemon $)=\lambda \theta_{L} / \theta_{S}<\lambda$ for $\lambda>0$. Let $x \equiv \theta_{L} / \theta_{S}<1$.

Note, with $\Delta \equiv \operatorname{prob}($ favorable $\mid$ star $)$ - prob(favorable $\mid$ lemon $), \frac{\partial \Delta}{\partial \lambda}=\lambda(1-x)>0$. An increase in $\lambda$ implies the test is more accurate because the difference between stars and lemons in the probability of a favorable signal is a positive function of $\lambda$, and because $\operatorname{prob}($ favorable $\mid$ star $)=1$ if $\lambda=1$.

Let $\sigma$ equal the fraction of stars in the population. As will be seen, we must derive the probability a T1 has hired a star, given a favorable signal, prob(star|favorable), and the probability a T2 has hired a lemon, given an unfavorable signal,

\footnotetext{
${ }^{1}$ The interview process is not modeled explicitly. For simplicity, it is assumed the signal is the same regardless of what type of school interviews an individual, and individuals are able to interview with both types of schools.

${ }^{2}$ Athey et al. (2007) find first year micro and macro grades are “...statistically significant predictors of student job placement, even conditional on $\mathrm{PhD}$ completion.” (p.512).
} 
prob(lemon|unfavorable). If $\lambda>0$, some lemons receive a favorable signal. If $\lambda=0$, no one receives a favorable signal. We have:

$$
\begin{aligned}
& \operatorname{prob}(\text { star } \mid \text { favorable })= \\
& \frac{\operatorname{prob}(\text { favorable } \mid \text { star }) \text { prob }(\text { star })}{\operatorname{prob}(\text { favorable } \mid \text { star }) \operatorname{prob}(\text { star })+\text { prob }(\text { favorable } \mid \text { lemon }) \operatorname{prob}(\text { lemon })}=\frac{\lambda \sigma}{\lambda \sigma+\lambda(1-\sigma) x}= \\
& \frac{\sigma}{\sigma+(1-\sigma) x},
\end{aligned}
$$

$\operatorname{prob}($ lemon $\mid$ unfavorable $\mid)=$

$$
\begin{aligned}
& \frac{\operatorname{prob}(\text { unfavorable } \mid \text { lemon }) \operatorname{prob}(\text { lemon })}{\text { prob(unfavorable|lemon) } \text { prob(lemon })+ \text { prob }(\text { unfavorable } \mid \text { star }) \text { prob }(\text { star })}= \\
& \frac{(1-\lambda x)(1-\sigma)}{(1-\lambda x)(1-\sigma)+\sigma(1-\lambda)} \text {. }
\end{aligned}
$$

Since $\lambda$ has the same impact on prob(favorable|star) and prob(favorable|lemon), it cancels out when deriving prob(star|favorable). Note, $\frac{\partial p r o b(\text { star } \mid \text { favorable })}{\partial \sigma}>0$, and $\frac{\partial \operatorname{prob}(\text { lemon|unfavorable })}{\partial \sigma}<0$. If the fraction of stars in the population, $\sigma$, falls, there is less likelihood of a star given a favorable signal and more likelihood of a lemon given an unfavorable signal. This fact is important as will be seen below.

Individuals are more likely to move from T1s to T2s than from T2s to T1s if T1s are more likely to hire lemons than T2 are to hire stars, or if:

$$
1 \text { - prob(star|favorable) > } 1 \text { - prob(lemon|unfavorable), }
$$

or if

$$
\text { prob(lemon|unfavorable })>\operatorname{prob}(\text { star|favorable }) \text {. }
$$


Thus, the likelihood of moving down exceeds the likelihood of moving up if T2s are more likely to hire lemons than T1s are to hire stars, and, as seen above, this possibility increases the smaller is $\sigma$.

Now prob(lemon|unfavorable) is positively related to $\lambda$, and equals one if $\lambda$ equals one. Also, prob(star|favorable) is independent of $\lambda$, and is less than one if $\sigma$ is less than one. Thus, $\operatorname{prob}($ lemon|unfavorable) $>\operatorname{prob}($ star|favorable) for all values of $\lambda$ if this inequality holds as $\lambda$ approaches zero. This occurs if:

$$
\frac{\sigma}{\sigma+(1-\sigma) x}<1-\sigma,
$$

or if

$$
\frac{1}{x}<\frac{(1-\sigma)^{2}}{\sigma^{2}}
$$

If stars and lemons exist in equal numbers, $\sigma=1 / 2$ and the RHS of ineq.(4') $=1$, so the inequality would not hold. If, as one might expect, there are fewer stars than lemons, then the inequality may hold. If $\sigma=.4$, the RHS of ineq.(4') $=2.25$, so, if $\theta_{S} / \theta_{L}<2.25$, the inequality holds. If $\sigma=.2$, the RHS of ineq.(4') $=16$, so $\theta_{S} / \theta_{L}<16$ is consistent with the inequality holding.

Unless $\theta_{S} / \theta_{L}$ is too large, if stars are less common than lemons $(\sigma<1 / 2)$, it is more likely there is a lower probability of moving up than there is of moving down. Thus, say $x=1 / 2=\lambda$, and $\sigma=.4$. Then $1-\operatorname{prob}($ star|favorable $) \approx .43$, and $1-\operatorname{prob}($ lemon|unfavorable) $\approx .31$. If $x=1 / 2=\lambda$ and $\sigma=.2$, $1-\operatorname{prob}($ star|favorable $) \approx .67$, and $1-\operatorname{prob}($ lemon|unfavorable $) \approx .14$. 
Again, the RHS of ineq.(4') reflects the smallest value for

prob(lemon|unfavorable). If there are fewer stars than lemons, ineq.(4') holds as long as $1 / x$ is not too large. Thus, it is quite plausible T1s make more "mistakes" than T2s do, so there is more movement from higher ranked schools down than there is from lower ranked schools up, as was found by Oyer (2008). ${ }^{3}$

To show $\sigma$ does not have to be small if $\lambda$ is not close to zero, consider the case

$$
\begin{aligned}
& \text { when } \lambda>0 \text { and } \sigma=1 / 2 \text {. If } \sigma=1 / 2 \text {, prob }(\text { star } \mid \text { favorable })=\frac{1}{1+x} \text {, and } \\
& \operatorname{prob}(\text { lemon } \mid \text { unfavorable })=\frac{1-\lambda x}{2-\lambda(1+x)} \text {. Now } \\
& \operatorname{prob}(\text { lemon } \mid \text { unfavorable })>\operatorname{prob}(\text { star } \mid \text { favorable }) \text { if: }
\end{aligned}
$$

$$
\lambda>\frac{1-x}{1-x^{2}} \equiv \lambda^{*}
$$

We have

$$
\frac{\partial \lambda^{*}}{\partial x}=2 x-x^{2}-1
$$

Now $\frac{\partial \lambda^{*}}{\partial x}$ is maximized when $x=1$ and $\frac{\partial \lambda *}{\partial x}=0$. For $x<1, \frac{\partial \lambda^{*}}{\partial x}<0$, so the larger is $x$ (the smaller is $\theta_{S} / \theta_{\mathrm{L}}$ ), the smaller is $\lambda^{*}$, and it will be more likely stars will move up than lemons will move down. For example, using ineq.(5), if $x=1 / 3, \lambda^{*}=3 / 4$, and, if $x=1 / 2$,

\footnotetext{
${ }^{3}$ Groothuis, Hill, and Perri (2007) consider what happens to the probability of finding high quality talent when the lower bound for high quality increases, talent is distributed continuously, and the signal a firm receives when it hires is the same as that used herein. They find, the higher the level of talent desired, the smaller the probability one with a favorable signal exceeds the threshold for high talent. This effect occurs for any continuous distribution of talent, and is simply due to the fact there are fewer individuals who exceed any threshold for high talent the larger the threshold.
} 
$\lambda^{*}=2 / 3$. Again, these results are for $\sigma=1 / 2$. Smaller values of $\sigma$ make it more likely we have more downward movement than upward movement.

\section{Conclusion}

Oyer $(2007,2008)$ found economics professors are more likely to move down from higher ranked schools than up from lower ranked schools. In this paper, it was demonstrated an asymmetric information model with screening may explain the observed behavior of these professors. The smaller the fraction, $\sigma$, of more able individuals (stars) in the population, the more likely are higher ranked schools to hire less able individuals (lemons), and the less likely are lower ranked schools to hire more able individuals. Thus a lower $\sigma$ implies a greater likelihood professors will move down to lower ranked schools at a greater rate than they will move up from lower ranked schools.

A more accurate testing mechanism $(\mathrm{d} \lambda>0)$ does not affect the probability of hiring a star given a favorable signal, but increases the probability of hiring a lemon given an unfavorable signal. If stars only receive a favorable signal $(\lambda=1)$, no stars would be hired in lower ranked schools, so these schools would make no mistakes and have no one to move up, but some lemons would still receive a favorable signal and be hired at higher ranked schools. Thus, in the sense, the more accurate the test, the less likely are stars to get an unfavorable signal, we find a more accurate test increases the likelihood upward movement will be less frequent than movement in the opposite direction. 


\section{References}

Athey, Susan, Katz, Lawrence F., Krueger, Alan B., Levitt, Steven, and Poterba, James. "What Does Performance in Graduate School Predict? Graduate Economics Education and Student Outcomes. American Economic Review 97 (May 2007): 512-518.

Groothuis, Peter, Hill, James Richard, and Perri, Timothy. "The Dilemma of Choosing Talent: Michael Jordans are Hard to Find.” Applied Economics 41 (November-December 2009): 3193-3198

Oyer, Paul. "Initial Labor Market Conditions and Long-Term Outcomes for Economists.” Journal of Economic Perspectives. 20 (Summer 2006): 143-160.

. “Is There an Insider Advantage in Getting Tenure?” American Economic Review 97 (May 2007): 501-505.

. "Ability and Employer Learning: Evidence from the Economist Labor Market.” Journal of the Japanese and International Economies 22 (June 2008): 268-289.

Smeets, Valérie, Warcynski, Frédéric, and Coupé, Tom. "Does the Academic Labor Market Initially Allocate New Graduates Efficiently?” Journal of Economic Perspectives 20 (Summer 2006): 161-172. 